\title{
Cholinergic and adrenergic control of heart-rate changes in the rabbit*
}

\author{
EMMANUEL KAZIS, S. DUNCAN \\ and D. A. POWELL \\ Neuroscience Laboratory, VA Hospital \\ Columbia, S.C. 29201 \\ and \\ University of South Carolina, Columbia, S.C. 29208
}

The effects of atropine and propranolol upon the heart rate (HR) of albino and pigmented rabbits were tested in a replicated Latin square design. (a) The unconditioned HR response to peripheral shock was usually an acceleration in rate, although on occasions decelerations were obtained. (b) There were no differences in the HR URs of pigmented and albino rabbits. (c) HR changes elicited by shock did not diminish over sessions. And (d) HR changes during drug conditions suggested that although sympathetic activation contributed to the HR UR in the rabbit, the release of vagal inhibition also accounts for a reliable proportion of this response.

Experiments on conditioned and unconditioned cardiovascular behaviors have revealed both increases and decreases in heart rate (HR) under various conditions (Obrist, Webb, Sutterer, \& Howard, 1972). A recent experiment revealed unconditioned heart rate (HR) increases in the rabbit to be jointly determined by the sympathetic and parasympathetic systems (Kazis, Milligan, \& Powell, 1973). In this study, separate groups of naive $\mathrm{Ss}$ were administered beta adrenergic and parasympathetic blockades and tested on a single day with four intensities of shock. These results agree with those of other investigators (e.g., Katcher, Solomon, Turner, LoLordo, Overmier, \& Rescorla, 1969; DeToledo \& Black, 1966). However, HR CRs have been both increases and decreases, depending upon the species, experimental conditions, etc. (e.g., Katcher et al, 1969: Schneiderman, VanDercar, Yehle, Manning, Goldem, \& Schneiderman, 1969; Lacey, 1967). On the other hand, Schneiderman et al (1969) obtained decelerative HR URs in the rabbit. The present experiment studied HR URs in the rabbit in more detail in an effort to ascertain under what conditions they might become accelerations or decelerations in rate. Preliminary results from a pilot experiment suggested that initial unconditioned heart rate accelerations might become decelerations as a function of repeated experimental sessions. Since in the original report of accelerative HR URs (Powell, Milligan, \& Kazis, 1970) naive Ss were used, the present experiment utilized a within-Ss design so that possible changes in HR topography might be assessed over sessions. Another

*This research was supported by VA Institutional Research Funds, Project No.5737-02. Requests for reprints should be mailed to D. A. Powell, Neuroscience Laboratory, VA Hospital, Columbia, S.C. 29201 possible source of variation involves strain differences. Consequently, two strains of rabbits, New Zealand pigmented and albino, were used in the present experiment.

\section{METHOD \\ Subjects and Apparatus}

Twenty pigmented and albino New Zealand rabbits weighing between 5 and $8 \mathrm{lb}$ served as Ss. The apparatus is described fully in Kazis et al (1973). Briefly, the Ss were run in a soundproof chamber while restrained in a Plexiglas restrainer. Shock and recording electrodes are also fully described in Kazis et al (1973).

\section{Procedure}

A Latin-square design was used in which two groups of five pigmented animals each received one of the five drug conditions. The drugs used were: (a) atropine sulfate $(25 \mathrm{mg} / \mathrm{kg})$, (b) propranolol hydrochloride (Inderal) $(10 \mathrm{mg} / \mathrm{kg})$, (c) atropine methyl nitrate $(25 \mathrm{mg} / \mathrm{kg})$, (d) propranolol $(10 \mathrm{mg} / \mathrm{kg})$ and atropine sulfate $(25 \mathrm{mg} / \mathrm{kg})$, or (e) propranolol $(10 \mathrm{mg} / \mathrm{kg})$ and atropine methyl nitrate $(25 \mathrm{mg} / \mathrm{kg})$. Thus the beta adrenergic receptors of the cardiovascular system were blocked by propranolol, while atropine was used to block the parasympathetic input to this system. Drug dosages were determined in prior experiments (Powell, Goldberg, Dauth, Schneiderman, \& Schneiderman, 1972; Kazis et al, 1973). All injections were subcutaneous and were made $15 \mathrm{~min}$ before the experiment began. The design was such that each drug treatment followed immediately after each of the other drugs once. All treatment days were separated by a saline day. In addition, each treatment animal was paired with a saline control animal run at the same time under identical conditions.

Ss of each group were administered four random presentations of each of four shock intensities, $0,1,3$, and $6 \mathrm{~mA}$. The shock duration was $1 / 2 \mathrm{sec}$ and was immediately preceded by a 5 -sec period during which baseline HR was recorded. The intertrial interval averaged $3 \mathrm{~min}$. The baseline $H R$ response measure consisted of the mean duration of 2 blocks of 5 beats each immediately preceding shock onset. Postshock measures were based on the 5-beat duration of 12 blocks of 5 beats occurring after shock onset. Percent changes were computed by dividing the difference between the pre- and each of the 12 postmeasures by the premeasure. Increases in HR (decreases in 5-beat durations) were arbitrarily assigned positive values. The above condiitons were replicated once using 10 albino New Zealand rabbits.

\section{RESULTS AND DISCUSSION}

The results showed the unconditioned HR response in most rabbits to be a short-latency but long-duration acceleration in rate, followed by a return to baseline, with the magnitude of the response being positively related to stimulus intensity. Only one animal showed clear-cut HR decelerations throughout the entire experiment, while another $\mathrm{S}$ initially showed a biphasic response consisting of a short-duration deceleration followed by a longer lasting acceleration with a return to baseline. However, this response later became monophasic as the animal began to show only HR accelerations during the latter half of the experiment. 


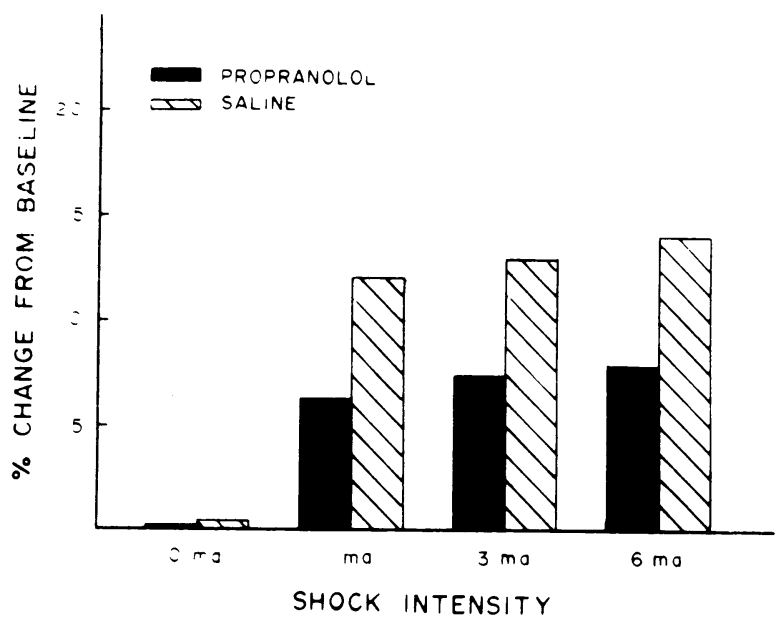

Fig. 1. Mean percent heart rate change in response to electric shock of rabbits treated with either saline or propranolol hydrochloride $(10 \mathrm{mg} / \mathrm{kg})$. The data represent the change in $R \cdot R$ durations of the second and third postshock 5-beat intervals compared to two blocks of 5 beats immediately preceding the shock $(\mathbf{N}=10)$.

Thus. the predominant HR UR in the chronic rabbit appears to be an acceleration in rate, although decelerations obviously occur in some animals.

In virtually all Ss, the second and third blocks of 5 beats showed the greatest change from baseline. Consequently, the mean percent change of these two blocks of 5 beats was subjected to further analysis. These data were analyzed by analysis of variance techniques in conjunction with tests of individual comparisons based upon a priori predictions of the response topography under different drug conditions (Kazis et al, 1973). This analysis revealed that the differences between the HR responses of pigmented and albino animals was not reliable. Therefore, further analysis of the data was based on the pooled results from the two groups. This analysis further revealed no adaptation of the absolute magnitude of HR URs over sessions in either the saline or drug groups.

The effects of beta-adrenergic blockade (propranolol) upon shock-elicited HR accelerations is shown in Fig. 1. As expected, propranolol blocked the normally occurring HR accelerations to electric shock. A small, but significant $(p<.01)$, increase still occurred, however. The unconditioned HR response under these conditions remained a short-latency long-duration acceleration, with a slow return to baseline after 12 5 -beat intervals. Figure 1 shows that the magnitude of the response under propranolol conditions was also positively related to shock intensity.

The effects of the parasympathetic blockades (atropine sulfate, $\mathrm{ASO}_{4}$ ) are shown in Fig. 2. This figure shows HR changes obtained on the atropine sulfate day compared with the saline day HR changes following the respective drug conditions. Both atropine sulfate and methyl atropine were shown to be significantly different from their saline control day HR changes $(p<.05$, $\mathrm{p}<.01$, respectively). However, the atropine drugs were not significantly different from each other $(p>10)$. Thus, methyl atropine data are not shown.

Blockade of the HR UR was virtually complete in the double blockade condition (propranolol-atropine sulfate and propranolol-methyl atropine). The typical unconditioned HR topography was not obtained under these conditions. Figure 3 shows the double blockade condition (atropine sulfate and propranolol) compared with the saline day which followed each treatment. Both double blockade conditions were significantly different from their saline conditions $(p<.01)$, but were not different from the HR changes elicited by zero shock $(\mathrm{p}>.10)$.

The results of the present experiment thus showed: (a) that the unconditioned HR response to peripheral shock in the chronically maintained rabbit is an acceleration in rate, although on occasion decelerations may also be obtained in some animals; (b) that there were no differences in the HR URs of pigmented and albino rabbits; (c) that HR changes elicited by shock do not diminish over sessions; and (d) that although sympathetic activation contributed to the HR UR in the rabbit, vagal inhibition also accounts for a reliable proportion of this response.

Unlike the HR CR in the rabbit, which is a pronounced slowing (Schneiderman et al, 1969), the HR UR in most animals was found in the present study to be an acceleration throughout the entire experiment. This confirms a prior experiment in which naive Ss were shown to exhibit unconditioned HR accelerations (Kazis et al, 1973). However, the finding that a few Ss (10\%) showed HR decelerations in response to shock suggests that the factors controlling unconditioned cardiovascular changes are not simple. For example, Schneiderman et al (1969) reported decelerative HR URs in the acutely

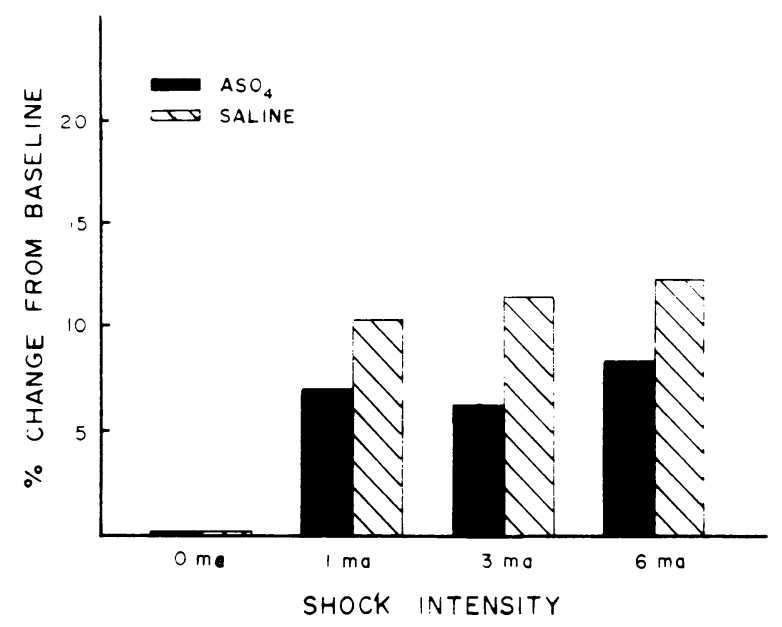

Fig. 2. Mean percent heart rate change in response to electric shock of rabbits treated with saline or atropine sulfate $\left(\mathrm{ASO}_{4}\right)$ $(25 \mathrm{mg} / \mathrm{kg})$. The data represent the change in $R \cdot R$ durations of the second and third postshock 5-beat intervals compared to blocks of 5 beats immediately preceding the shock $(N=10)$. 
prepared albino rabbit. The results of the latter experiment suggested that blood pressure increases may importantly influence the directionality of both HR CRs and URs. Katcher et al (1969) have also made this point with respect to the control of conditioned and unconditioned HR changes in the dog. Thus, in individual animals such homeostatic mechanisms may become operative at different times and under different conditions. Therefore, definitive studies of HR should simultaneously make measurements of or manipulate these mechanisms to assess their effects on HR changes.

The present results also confirm the findings of Kazis et al (1973) that HR accelerations elicited by peripheral shock in the rabbit are jointly determined by the sympathetic and parasympathetic inputs to the heart. Thus the tests of individual comparisons showed all drug conditions to differ reliably from their respective saline control conditions. Likewise, although the atropine and propranolol differences were not significant, both were significantly different from the double blockade

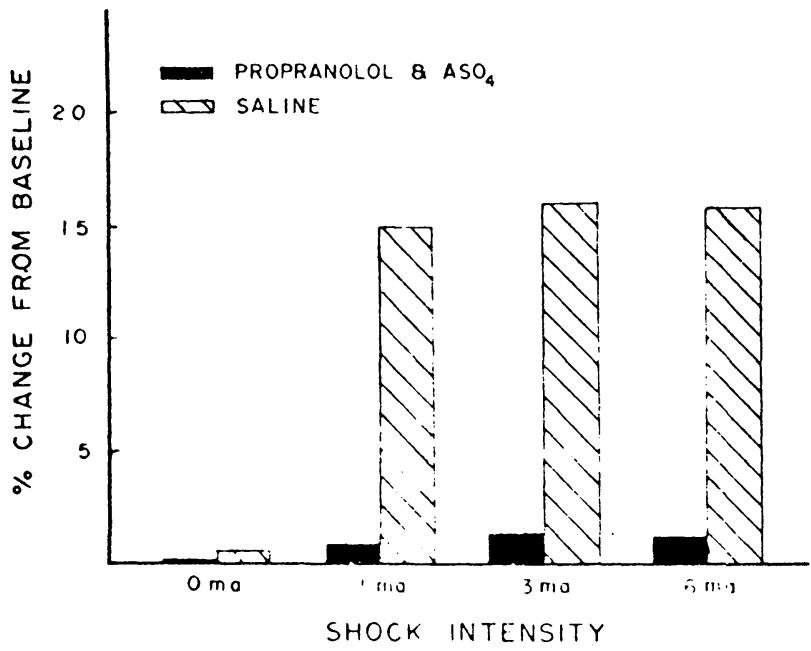

Fig. 3. Mean percent heart rate change in response to electric shock of rabbits treated with either saline or propranolol hydrochloride $(10 \mathrm{mg} / \mathrm{kg})$ and atropine sulfate $(25 \mathrm{mg} / \mathrm{kg})$. The data represent the change in $R-R$ durations of the second and third postshock 5-beat intervals compared to two blocks of 5 beats immediately preceding the shock $(\mathrm{N}=10)$. conditions, but the latter were not reliably different from the nonshock conditions. These tests thus show that both beta adrenergic blockade (which blocks the sympathetic accelerative input to the heart) and cholinergic blockade (which blocks the parasympathetic decelerative inputs to the heart) produce decrements in the normally occurring HR accelerations to electric shock. Cohen \& Pitts (1968) and Dykman \& Gantt (1959) have reported that a similar dual control of HR changes exists in the pigeon and dog, respectively. Powell et al (1972) and Kazis et al (1973) have also recently reported cholinergic and adrenergic interactions in the control of the rabbit's HR CR to peripheral shock and the UR elicited by electrical brain stimulation.

\section{REFERENCES}

Cohen, D. H. \& Pitts, L. H. Vagal and sympathetic components of conditioned cardio-acceleration in the pigeon. Brain Research, $1968,9,15-31$

DeToledo, L., \& Black, A. H. Heart rate: Changes during conditioned suppression in rats. Science, 1966, 152, 1404-1406.

Dykman, R. A., \& Gantt, W. H. The parasympathetic component of unlearned and acquired cardiac responses. Journal of Comparative \& Physiological Psychology, 1959, 52, 163-167.

Katcher, A. H., Solomon, R. L., Turner, L. H., LoLordo, V., Overmier, J. B., \& Rescorla, R. A. Heart-rate and blood pressure responses to signaled and unsignaled shocks: Effects of cardiac sympathectomy. Journal of Comparative \& Physiological Psychology, 1969, 68, 163-174.

Kazis, E., Milligan, W. L., \& Powell, D. A. Autonomic-somatic relationships: Blockade of heart rate and corneo-retinal potential responses. Journal Comparative \& Physiological potential responses. Journal

Lacey, J. I. Somatic response patterning and stress: Some revisions of activation theory. In $M$. H. Appley and $R$. Trumbull (Eds.), Psychological stress: Issues in research. New York: Appleton-Century-Crofts, 1967. Pp. 14-42.

Obrist, P. A., Webb, R. A., Sutterer, J. R., \& Howard, J. L. The cardiac-somatic relationship: Some reformulations. Psychophysiology, 1970, 6, 569-587.

Powell, D. A., Milligan, W. L., \& Kazis, E. Divergencies in the conditioned and unconditioned heart-rate response in the rabbit. Proceedings of the 78 th Annual American Psychological Association, 1970, 5, 257-258.

Powell, D. A., Goldberg, S., Dauth, G. W., Schneiderman, E., \& Schneiderman, N. Adrenergic and cholinergic blockade of cardiovascular responses to subcortical electrical stimulation in unanesthetized rabbits. Physiology \& Behavior, 1973, 8, 927-936.

Schneiderman, N. E., V anDercar, D. H., Yehle, A. L., Manning, A. A., Golden, T., \& Schneiderman, E. Vagal compensatory adjustment: Relationship to heart rate classical conditioning in rabbits. Journal of Comparative \& Physiological Psychology, 1969, 68, 175-183.

(Received for publication September 21, 1973.) 